\title{
Les délits de monnaie dans les provinces basques (1551-1700) : une criminalité de l'étranger?
}

\section{Olivier Caporossi}

\section{(2) OpenEdition}

\section{Journals}

\section{Édition électronique}

URL : http://journals.openedition.org/abpo/1025

DOI : 10.4000/abpo. 1025

ISBN : 978-2-7535-1517-8

ISSN : 2108-6443

\section{Éditeur}

Presses universitaires de Rennes

\section{Édition imprimée}

Date de publication : 20 avril 2010

Pagination : 223-239

ISBN : 978-2-7535-1146-0

ISSN : 0399-0826

\section{Référence électronique}

Olivier Caporossi, «Les délits de monnaie dans les provinces basques (1551-1700) : une criminalité de l'étranger?", Annales de Bretagne et des Pays de l'Ouest [En ligne], 117-1 | 2010, mis en ligne le 20 avril 2012, consulté le 19 avril 2019. URL : http://journals.openedition.org/abpo/1025 ; DOI : 10.4000/ abpo. 1025

Ce document a été généré automatiquement le 19 avril 2019

(c) Presses universitaires de Rennes 


\title{
Les délits de monnaie dans les provinces basques (1551-1700) : une criminalité de l'étranger?
}

\author{
Olivier Caporossi
}

1 Depuis le règne de Charles Quint (1516-1556), l'afflux régulier des métaux précieux américains (or et surtout argent) permet au roi catholique d'acheter en bonne monnaie clientèles et alliances, sans oublier de payer le tercio engagé dans la guerre des Flandres (1568-1648) ${ }^{1}$. Dès le XvI ${ }^{\mathrm{e}}$ siècle, la question monétaire place les trois provinces basques (le Guipuzcoa, la Biscaye et le pays d'Alava) au cœur des relations commerciales, militaires et politiques entre l'Espagne, la France, les îles Britanniques et les Pays-Bas, comme le montrent différentes études menées à partir des sources douanières de l'Ancien Régime². La multiplication des conflits et des champs d'opération comme les banqueroutes successives de la monarchie hispanique $(1557,1575,1596)$ aboutissent à la mise en place d'une nouvelle politique monétaire, celle du billon, dont la valeur intrinsèque déjà peu importante ne cesse d'être dévaluée. Créée en 1599, la monnaie de billon est refrappée au double de sa valeur en 1604. Cette politique, contestée de toute part, est le contexte dans lequel se déploie le faux-monnayage. Les dévaluations monétaires se multiplient : celle de $50 \%$ en 1628 est suivie d'autres dévaluations en 1634, 1636, 1641, 1642 et 1664 . La réforme monétaire de 1680 se traduit par une chute du billon, qui perd les trois quarts de sa valeur, et les réaux d'argent sont à leur tour dévalués de $20 \%$ en 1686. En réalité, la politique monétaire de la monarchie catholique, qui impose un système complexe fondé sur une nouvelle monnaie intérieure, le billon, ouvre une longue série d'altérations monétaires et de dévaluations opérées pour financer la guerre contre Louis XIII et Louis XIV (1635-1659, 1667-1668, 1672-1678, 1683-1684, 1690-1697) ${ }^{3}$, l'Angleterre d'Élisabeth I $^{\text {re }}$ (1584-1603), de Charles I ${ }^{\text {er }}$ et d'Olivier Cromwell (1625-1630 et 1655-1659) et les rebelles hollandais (1566-1609, 1621-1648) avant que la réforme de Rodrigo Caballero (1706-1716) ne puisse stabiliser le système des deux circulations monétaires ${ }^{4}$. La bonne monnaie, faite des métaux américains, fuit ainsi à l'étranger et se trouve remplacée par de la fausse monnaie introduite dans l'économie espagnole par les marchands français, anglais ou 
hollandais. Or, la crise monétaire entraîne le faux monnayage dès lors que les États décident un cours des espèces différent de leur valeur intrinsèque, permettant aux faussaires d'envisager un bénéfice de la différence entre ces deux valeurs ${ }^{5}$.

2 La politique monétaire de Louis XIII qui, pour financer la guerre contre la maison d'Autriche à partir de 1636, achète le doublon d'or espagnol à 10 livres tournois, soit $25 \%$ de plus que le prix du marché ${ }^{6}$, et l'investissement des Hollandais dans la fabrication de faux billons, destinés à acheter illégalement la bonne piastre dans les contreforts pyrénéens et les littoraux atlantiques de l'Espagne pour la revendre aux officiers de la maison des monnaies de Bayonne ou de Bordeaux ${ }^{7}$, comme la dénonciation de l'emprise des marchands français et flamands sur l'économie castillane, font craindre une mainmise de l'ennemi sur les ports ibériques de la côte atlantique. La criminalité monétaire, dont les sources littéraires, administratives et judiciaires du XVII ${ }^{e}$ siècle nous livrent un portrait multiple (contrebandiers de monnaie, fabricateurs, distributeurs, rogneurs, billonneurs), implique la figure de l'étranger au royaume et au roi. Soupçonné d'usurper le ius monetae du roi catholique, jusqu'à ouvrir la possibilité d'une qualification du crime de monnaie en crime de lèse-majesté, tant par le viol de la figure sacrée du monarque que par la menace ainsi produite à l'encontre de l'harmonie des sociétés hispaniques, l'étranger devient la qualification définitoire de cette délinquance particulière. C'est, sans doute, par ce biais que l'institution monarchique cherche à construire la figure de l'ennemi. La seule qualification d'étrangeté du crime de monnaie n'y suffit pas. La répression du crime de monnaie, comme l'un des principaux modes de criminalisation de l'étranger dans l'Espagne des derniers Habsbourg, s'inscrit dans un espace concret, capable d'identifier les enjeux frontaliers d'une telle démarche aussi bien judiciaire que politique et d'en promouvoir les acteurs comme les promoteurs: les trois provinces basques.

3 Le frère Pedro de Oña (1560-1626), évêque de Gaeta depuis 1605, dénonce l'usage monétaire des étrangers dans la péninsule ibérique ${ }^{8}$. Les étrangers sont accusés d'introduire de la fausse monnaie de billon pour l'échanger contre de la bonne monnaie d'or et d'argent qu'ils rapportent chez eux. Les ports de Castille et plus encore ceux de Biscaye représenteraient les portes par lesquelles la fausse monnaie étrangère pénètrerait dans la péninsule. La complicité des élites locales (l'auteur dénonce autant les habitants de ces contrées que les gardes-frontières, et les gardes des puertos secos) expliquerait le succès de l'introduction de la fausse monnaie étrangère dans l'économie espagnole. Dans sa Restauración política de España éditée dès 1619, le donneur d'avis tolédan Sancho de Moncada stigmatise le commerce du faux billon des étrangers présents en Biscaye et la sortie de la monnaie d'argent au profit des Français ${ }^{9}$. Vers 1628 le docteur Sebastián Benítez Negrete voit dans les ports atlantiques les meilleures portes d'entrée du faux billon et de la contrebande monétaire des étrangers hostiles à la monarchie catholique $^{10}$.

4 Pour la cour d'Espagne c'est l'ensemble des provinces basques, du fait de leur statut fiscal et de leur situation stratégique entre la France, la Navarre et la Castille, qui représente la plaque tournante de la contrebande monétaire et du faux monnayage atlantique. En décembre 1607, de Paris, Rodrigo de Cordoba informe le duc de Lerma, favori du roi Philippe III jusqu'en 1616, sur la réalité des cargaisons de faux billons embarquées par des marchands anglais, français et hollandais dont la flotte prétend venir d'Hambourg et de Lubeck $^{11}$. Dès 1609 la monarchie reçoit des avis sur l'existence, dans le Sud Ouest de la France, de quatre ateliers spécialisés dans la refonte de la monnaie d'argent sortie 
illégalement d'Espagne ${ }^{12}$. Le conseiller Francisco de Tejada dénonce à Philippe IV dans une consulte du 8 février 1622, le rôle des rebelles hollandais dans la contrebande de faux billons, associant l'étranger au royaume à l'hérésie et au faux monnayage ${ }^{13}$. Lorsque la paix entre les monarchies chrétienne et catholique semble revenue, après le traité des Pyrénées (1659), et que le conseil de Castille se voit obligé de consulter le roi sur les excès des Français dans la province de Guipúzcoa, ce sont les délits monétaires de ces derniers qu'il met en avant. Dans un rapport adressé au conseil de Castille, le corregidor du Guipúzcoa, Martin Joseph Badaran, dénonce la menace que fait peser sur la sécurité de la frontière basque, l'intrusion d'une bande de dix-sept à vingt Français munis d'armes à feu qui prit d'assaut la prison d'Usúrbil où était détenu sur ordre de l'alcade un de leurs compatriotes accusé d'introduire de la fausse monnaie dans la péninsule. La figure de l'étranger faux-monnayeur semble imprégner la correspondance des conseillers du roi, des corregidors et des diplomates pendant tout le siècle. Elle suscite autant qu'elle justifie une législation plus sévère envers les auteurs des crimes de monnaie. Philippe IV (1621-1665) élargit les facilités probatoires établies par Philippe II en 1598 pour les cas de sodomie et d'hérésie, en permettant aux juges royaux de prouver les délits de monnaie avec les seules déclarations de trois témoins, fussent-ils les complices de l'accusé. Cette pragmatique de 1636 est renforcée dès 1637 par une nouvelle disposition royale qui dote le conseil royal des capacités légales pour traiter des crimes de monnaie par des procédures d'exception privilégiant une certaine pratique de l'arbitraire, la vía de gobierno 14 .

5 Les provinces basques et leurs ports constituent donc le laboratoire de cette nouvelle politique répressive qui s'inscrit dans la mise en place progressive d'une police des étrangers dont témoigne la multiplication des registres à l'échelle locale (comme à SaintSébastien) ou à l'échelle du royaume ${ }^{15}$. La mesure de cette répression ne saurait être prise à la lumière des archives judiciaires (procès d'appel ou réservés) ou administratives (consultes et rapports) du seul conseil de Castille, qui lorsqu'il agit comme cour suprême de la monarchie catholique, prend le titre de conseil royal. C'est pourquoi il nous a paru intéressant d'observer en quoi les procédures pénales du grand juge de Biscaye (juez mayor) répondent ou non aux nouvelles exigences des Habsbourg contre la criminalité monétair ${ }^{16}$. Sur les 22 cas recensés parmi les procès pénaux et civils de la chambre de Biscaye (qui siège auprès de la chancellerie de Valladolid) ${ }^{17}$ pour la période 1551-1796, six relèvent du $\mathrm{XVI}^{\mathrm{e}}$ siècle et treize du XVII ${ }^{e}$. La question de l'étrangeté du crime de fausse monnaie y est posée de manière diverse et montre pour le moins le rôle de l'institution judiciaire dans les différents degrés de la fabrique de l'étranger au roi catholique.

\section{Le grand juge et la main de l'étranger}

Rares sont les procédures qui mettent directement en cause des étrangers de passage ou installés à Bilbao. Les ministres du grand juge de Biscaye, dont la charge est généralement assumée par le corregidor de cette province ${ }^{18}$ - que le roi nomme pour quatre années environ sur avis du conseil de la Chambre de Castille - n'en sont pas moins préoccupés par la présence de l'étranger, même lorsque les accusés sont des naturels ou d'autres sujets du roi catholique (Navarrais, Castillans, Aragonais etc.). Les tensions entre les chancelleries d'Europe se traduisent, en ce début du siècle, par l'envoi de commissaires pour surveiller les juges des douanes (alcaldes de sacas), contrôler les ports et lutter contre la contrebande monétaire. Le regard de Madrid se porte sur ces régions dont la 
population, très attachée à la défense de ses fors, conteste le bien fondé des différentes altérations monétaires qui se succèdent. Les résultats mitigés de ces commissaires s'expliquent par la difficulté qu'ils rencontrent à susciter une répression accrue des autorités locales contre la criminalité monétaire. Le tribunal de la chambre de Biscaye, préoccupé par la défense de sa juridiction, n'échappe pas à cette réticence. Par exemple, en 1674, l'un des magistrats de la chambre de Biscaye, Santiago de la Toba, est condamné à une amende de 2000 ducats pour avoir transmis au conseil royal les arrêts originaux relatifs à une procédure pénale pour fausse monnaie effectuée à Bilbao, contrevenant ainsi aux fors de la seigneurie de Biscaye ${ }^{19}$. L'intervention de la reine mère et des conseillers royaux produit un conflit de compétence qui scandalise les autorités provinciales mais permet d'annuler la première condamnation du greffier. Nous pouvons cependant constater que six des treize procès sont faits pendant les vingt premières années du siècle, au moment où le débat sur le faux billon est le plus intense et où le conseil de Castille cherche à développer son emprise sur cette frontière littorale.

7 La chambre de Biscaye n'échappe donc pas aux pressions de la cour et oriente une part importante de ses procédures contre la criminalité monétaire vers la recherche de la main de l'étranger. Derrière chaque arrestation pour fausse monnaie se cache peut-être une entreprise de l'Étranger. En 1610, deux habitants de Bilbao, Domingo de Leniz et Juan de Soto, sont poursuivis par le grand juge pour avoir introduit des faux billons dans les ports de Biscaye. La procédure de première instance menée par le licencié Juan de Carrion, qui reçut pour cela une commission privative du grand juge, met en avant les liens commerciaux réguliers existants depuis plusieurs années entre les accusés et un marchand français Anton de Urdanqui pour expliquer l'abondance de la fausse monnaie distribuée $^{20}$. Le lieu du crime est la demeure d'Antona de Vedia à Bilbao, où les deux compères et leurs complices avaient l'habitude de se loger et de conclure leurs affaires avec de mystérieux marchands français. Ils y auraient par exemple acheté du charbon avec des faux doublons de quatre réaux fournis par ces Français pour les revendre contre cinquante vrais réaux dans d'autres lieux de la Biscaye. La disparition de leurs prétendus complices fait de Domingo de Leniz et de Juan de Soto les seuls coupables. Le premier est condamné en première instance à quatre années de bannissement de la ville de Bilbao et à une amende de 100000 maravédis à payer à la justice, le second perd son office, est banni du royaume de Castille pour 20 ans et devra payer 250000 maravédis. Le procès en appel devant le grand juge leur permet néanmoins d'obtenir une réduction des sentences. Le 29 novembre 1611, Domingo de Leniz voit sa peine de bannissement confirmée mais pas son amende ; il en est absous. Le 8 octobre 1610 Joan de Soto voit sa peine révisée : il perd son office de procureur du roi mais son bannissement du royaume est limité à dix ans et son amende diminuée de 100000 maravédis. Pour la chambre de Biscaye, la découverte de faux doublons attribués à Domingo de Leniz et Juan de Soto fait de leur activité commerciale un paravent pour cacher la diffusion de fausse monnaie commanditée à partir de la France. La fausse monnaie est l'arme de guerre de l'ennemi, malgré la paix apparente qui règne entre les monarchies catholique et chrétienne.

La même suspicion se retrouve dans les poursuites établies par le licencié Francisco de la Puente, corregidor et grand juge de Biscaye, dès le 3 octobre 1619 contre un habitant de Bermeo, Pedro de Legorraza et un certain Juan de Soto. L'alcade ordinaire de Bermeo rend compte au grand juge des rumeurs qui circulent dans sa cité sur la diffusion de monnaies suspectes car d'aspect neuf, elles afficheraient la date de 1612, voire d'autres dates plus anciennes encore. Ces rumeurs de fausse monnaie se seraient répandues dans 
toute la seigneurie, troublant l'ordre public. Dans le bref récit qu'en donne la chambre de Biscaye, elles manifestent, aux yeux des échevins qui en appellent à une entité supérieure - celle du grand juge - la crise qui toucherait aussi bien la province que l'ensemble de la péninsule ibérique. Comparable à une peste sociale, la fausse monnaie "étrangère " remet en cause une regalia et de ce fait, l'obéissance des sujets de toute la province à leur souverain légitime. Toute une hiérarchie sociale et politique semble menacée, d'autant plus que son introduction, par la transgression des valeurs qu'elle promeut, est souvent considérée comme une remise en cause de la propriété et des biens de l'ensemble des sujets de la monarchie catholique. Quelques pièces de monnaie pourraient provoquer la destruction de la communauté.

9 Un deuxième bruit justifie l'établissement d'une procédure pénale : certaines personnes auraient fait passer de la monnaie d'or et d'argent en France et transporter des cuartos (pièces de billon de 4 maravédis) vers l'Espagne. Cette seconde rumeur, parce qu'elle confirme la nature «étrangère » des faux billons déjà annoncée dans la précédente, est une première réponse à l'impuissance manifestée par les autorités de Bermeo face au phénomène dénoncé, l'invasion de la Biscaye par la fausse monnaie venue de France. Elle donne à comprendre l'incompréhensible, et oriente la répression judiciaire vers l'anormalité monétaire du caractère étranger des pièces, et non par exemple vers un défaut de valeur intrinsèque (poids en métal précieux). L'objet du crime dirige la persécution judiciaire contre un ennemi surpuissant, la main de l'étranger dont la fausseté des monnaies serait l'incontestable signe.

L'auteur de l'enquête, le procureur du grand juge, Juan de Soto, cherche à identifier ces personnes parmi celles qui sont connues pour voyager souvent entre la France, les provinces basques, la Navarre et la Castille. Ils reçoivent des dénonciations qui leur permettent d'imputer le crime de fausse monnaie à un habitant de Bermeo, Pedro de Legorraza. Un des témoins interrogés, Domingo de Alestra dénonce l'accusé et prétend l'avoir vu à Vitoria chez un certain Lucas de Charte. C'est là que Pedro de Legorraza aurait reçu des marchandises à transporter jusqu'à Bilbao. Il aurait été vu par d'autres témoins avec deux sacs pesant chacun six arrobes (69 kilos environ) destinés à Doña Catalina de Butron, épouse d'un habitant de Bilbao, Pedro de Sabugal. Près de 1300 réaux se cacheraient dans ces mystérieux sacs. Arrêtés puis interrogés à Bilbao le 3 octobre 1619, Catalina de Butron et Pedro de Sabugal admettent connaître l'accusé mais nient que la fille d'un premier mariage de Pedro de Sabugal l'ait accueilli deux semaines auparavant, de nuit, dans leur demeure. L'inspection de la demeure de Pedro de Sabugal apporte alors de nouveaux éléments à la justice. Elle permet la découverte d'un coffre caché sous du linge blanc dans l'une des salles arrière de la maison. Une fois ouvert, ce dernier révèle la présence de petits sacs de toile pleins de pièces de monnaie dont la valeur est estimée à 200 réaux. Ces petits sacs mélangent cuartillos (pièces de 8 maravédis et demi) et cuartos (pièces de 4 maravédis). Face à cette nouvelle situation, le couple de prévenus reconnaît avoir reçu du principal accusé, Pedro de Legorraza, l'équivalent de 70 réaux. Dans l'interrogatoire des témoins par le procureur Juan de Soto, qui suit la procédure, l'absence des 1300 réaux de Pedro de Legorraza, n'empêche en rien le développement du récit judiciaire sur les faits et gestes du prétendu faux-monnayeur et, désormais, de ses complices. La fausse monnaie n'est pas définie par Juan de Soto comme une transgression de sa valeur intrinsèque (son poids en métaux précieux) mais comme la violation du droit royal de battre monnaie. L'expertise des quelques monnaies rencontrées chez Catalina de Butron et Pedro de Sabugal se fonde d'ailleurs plus sur la qualité de la frappe et son 
apparence extérieure que sur sa contenance. Toute monnaie autre que celle frappée dans les ateliers monétaires du roi est interdite, affirme Juan de Soto aux témoins qu'il interroge. À partir de la reconnaissance de ce prédicat, les différents témoins sont amenés à se prononcer sur son récit judiciaire : Pedro de Legorraza aurait apporté de Saint-Jean-de Luz à Bermeo puis à Bilbao d'importantes quantités de faux billons qu'il aurait mélangés avec de la vraie monnaie issue des ateliers royaux de Ségovie pour effectuer divers achats. Il aurait ensuite continué ces mêmes opérations de dissimulation avec la complicité d'un jeune serviteur, à Vitoria, Guernica et Saint-Sébastien. Il aurait aussi utilisé sa fausse monnaie pour rembourser quelques prêts contractés dans ces mêmes villes.

11 La main de l'étranger (et le sceau monétaire qui l'incarne) établit dans l'esprit des magistrats la culpabilité de leurs complices dont les tribunaux royaux ont du mal à repérer les activités criminelles. La force de destruction qui lui est attribuée n'est pas toujours suffisante pour discerner les signes de leur culpabilité souvent assimilés à la mobilité et à d'éventuels voyages en France ou de possibles rencontres avec des étrangers. Dès que l'appareil judiciaire cherche à renforcer la criminalisation de certaines activités monétaires, il lui faut hisser la répression à une autre hauteur. La recherche de "signes victimaires " dans l'anormalité des étrangers ${ }^{21}$, définie par un comportement commun fondé sur la mobilité géographique, l'instabilité sociale, et l'économie de la contrebande, aboutit à en faire une catégorie sociale, c'est-à-dire qu'elle permet à l'Institution judiciaire de mettre ensemble une série d'individus dont la surveillance et la répression constituent l'esquisse d'une véritable police.

\section{Une police des étrangers?}

La chambre de Biscaye juge des étrangers inculpés d'un crime de monnaie dès la seconde partie du XVI e siècle. Elle procède par exemple contre Olivier Lux pour contrebande de monnaie d'or, d'argent et de cuivre en $1551^{22}$. Trois années plus tard c'est un soldat français ayant servi dans la compagnie du capitaine Diego de Carrabajal en Guipúzcoa, Jean Amiot, qui est accusé de contrebande monétaire. En 1554, Jean Amiot est poursuivi au pénal par le juge de commission Domingo de Jauregui qui l'accuse de faire sortir d'Espagne des monnaies d'argent cachées dans sa chemise en cherchant à s'embarquer au port de Fontarabie, et surtout, d'être le complice d'un autre Français, Oñate Beja, arrêté avec 126 réaux à Mungia, malgré une violente résistance ${ }^{23}$. C'est dans le cadre d'une procédure civile, qu'en 1594, le procureur mandaté par la chambre de Biscaye, Juan Fernández Angulo, inculpe l'irlandais Enrique Taller d'exportation illégale de monnaie, sur plainte d'un tiers ${ }^{24}$. Le capitaine de navire, originaire de Diolin, d'abord soupçonné de vouloir porter en Irlande des quantités de monnaie introuvables, est finalement absous des différentes accusations qui pesaient sur lui par le licencié Gómez de la Puerta, grand juge de Biscaye, le 23 septembre 1594. Philippe II soutient alors ouvertement le soulèvement d'Hugh O'Neill (1594-1603) et prépare une nouvelle armada pour secourir ses alliés catholiques ${ }^{25}$. En 1589 déjà, des communautés irlandaises issues d'une émigration récente étaient installées sur la côte atlantique de l'Espagne: de SaintSébastien et Bilbao à Colindres, Santander, Viveros, El Ferrol et La Corogne ${ }^{26}$. En 1599, Philippe III finira par ordonner au corregidor de la seigneurie de Biscaye d'empêcher que les navires étrangers embarquent des marchandises pour les revendre dans un port 
ennemi, tout en prenant soin de supprimer la clause qui obligeait ces étrangers à déposer une caution, comme le réclamait la junte générale de Biscaye ${ }^{27}$.

Les périodes de conflit, même si elles dominent les $\mathrm{XVI}^{\mathrm{e}}$ et $\mathrm{XVII}{ }^{\mathrm{e}}$ siècles hispaniques, ne sont pas les seules à susciter recherches, arrestations et procès contre la criminalité monétaire des étrangers de passage en Espagne. Après la découverte de fausse monnaie sur leurs navires, la chambre de Biscaye juge les Français Pedro et Guillermo Pedame entre 1601 et $1602^{28}$. En 1616, c'est un autre Français, le nantais Juan Baleton, âgé de 21 ans, qui est poursuivi pour des délits comparables ${ }^{29}$. En première instance, la procédure pénale est menée par l'alcade ordinaire de Portugalete qui, le premier, établit les faits délictueux avant de nommer un curateur à l'accusé, considéré comme mineur par la loi castillane. Juan Baleton serait arrivé de Nantes à Bilbao avec le navire français de Pedro Baneos un mois avant le début du procès. Portugalete est le port où Français et Anglais viennent acheter du fer contre de la monnaie d'or, dont la sortie hors d'Espagne est conditionnée par l'obtention d'une licence royale. Il se serait rendu à Bilbao, où il se fit enregistrer comme marchand étranger venu en cette ville pour vendre deux besaces (dont l'une est d'Angoulême). Une fois la vente conclue, il tente de rejoindre clandestinement les deux navires de Pedro Baneo, dont il semble être le représentant, pour faire sortir illégalement d'Espagne la monnaie qu'il possède, soit environ 74 doublons (liards) et demi selon l'alguazil de ville qui les découvre dans l'un de ses paquets. Pedro Baneo, lui, réussit à s'enfuir avec ses deux navires. Il est condamné par contumace pour absence de licence et refus d'inspection de son navire, à la perte de tous ses biens en Biscaye alors que Juan Baleton, désormais prisonnier, doit, lui, payer une amende de 74 doublons à l'alcade ordinaire. Suite à la pétition envoyée à la chambre de Biscaye à la demande du facteur nantais, le grand juge inhibe l'alcade de Portugalete et une fois la sentence en appel confirmée, exige de ce dernier la restitution des 74 doublons.

La chambre de Biscaye ne limite pas son activité à la seule répression de la contrebande monétaire. Elle s'attaque parfois à de véritables faux-monnayeurs venus de l'étranger. L'arrêt du corregidor de Bilbao, daté du 9 mai 1698, dénonce la présence de fausse monnaie dans la capitale provinciale ${ }^{30}$. Une habitante de Bilbao reçoit d'une veuve d'Antequera, quatre écus d'argent pour l'achat de divers objets, mais méfiante, elle les fait expertiser par un orfèvre de sa connaissance, Domingo de Barendica, qui les déclare faux ; devant l'alguacil Juan de Zugasti, il explique que ces écus sont contrefaits, contiennent bien moins d'argent que la loi royale ne l'exige, n'ont pas été frappés dans un atelier royal et sont d'un poids trop léger pour être de la vraie monnaie. Le témoignage de la veuve Magdalena de Ascoabica permet ensuite de reconstituer, sous l'autorité judiciaire du corregidor de Bilbao, le parcours effectué par les quatre écus contrefaits et d'arrêter un suspect, le français Juan de Uriarte. Celui-ci, originaire de Lequeitio en France, est accusé d'être à la tête d'une véritable entreprise criminelle, avec cinq complices basques: Mariana de Gavica de Ascoa, Joan de Alboitiz et son épouse Maria Saenz de Barainca, Joan de Alboitiz Varanca, leur fils, et Mathias de Mutio. Juan de Uriarte est accusé de profiter de sa profession de coupeur dans une boucherie de Bilbao pour diffuser des fausses piastres de huit réaux, qu'il aurait rapportées de France, puis il est condamné en première instance à quatre années de galères et au bannissement perpétuel de la Biscaye, alors que ses complices supposés perdent leurs biens. Enfermé dans la prison de la ville de Bilbao, il fait appel de cette décision judiciaire devant le grand juge de Biscaye dès le 14 novembre 1698 . Le nouveau procès qui s'ensuit lui permet de présenter de nouveaux 
témoins, dont un Français d'Ustaritz, Bernardo de Echarrutua, qui certifie que le prévenu vit de l'aumône des autres et d'argumenter que les faux écus, difficiles à reconnaître, ont été reçus par sa femme, qui de par sa nature, ne pouvait les identifier comme de la fausse monnaie et les aurait reçus de bonne foi. Le grand juge finit par révoquer la première sentence, libérant Juan de Uriarte sans frais de justice, et lui restituant ses biens séquestrés par la justice du roi. Cette sentence est confirmée par la chancellerie de Valladolid le 10 avril 1699.

Ces quelques exemples appellent plusieurs remarques sur la nature de la police des étrangers que pratique le grand juge de Biscaye pendant près d'un siècle et demi. Le grand juge se montre plus intéressé à réprimer les Français que les autres communautés étrangères de la province. Comme corregidor il est l'homme du roi en Biscaye et se doit d'adhérer aux présupposés de la cour sur la police des étrangers. Mais, dans le même temps, l'exercice judiciaire de cette police qu'il assume demeure assez limité car il sait l'importance du commerce avec les Anglais et surtout avec la France pour la prospérité de la Biscaye. L'identité des prévenus laisse émerger la préférence des autorités pour des personnes dont le métier et le parcours professionnel montrent un certain nomadisme, c'est-à-dire une faible intégration dans la société basque. Jean Amiot est un soldat démobilisé qui tente de se reconvertir dans le commerce ; Enrique Taller est un capitaine de marine ; Juan Baleton est un représentant de commerce ; Juan de Uriarte est présenté comme un aide boucher instable : il travaille d'abord dans la province de Guipúzcoa au service du boucher Phelipe de Urrechurti, qu'il quitte au bout de huit ans, il devient alors le laquais de Joseph de Varasti Portejo à Vergara pendant trois ans avant de s'installer à Marquina au service d'un autre boucher durant trois autres années. Pendant les deux années qui suivent, il aurait erré en Biscaye à la recherche d'un nouvel emploi avant de s'installer à Lequeitio.

Leur arrestation ne soulève donc dans la plupart des cas aucune véritable résistance sociale. Quant à l'affaire Juan de Uriarte, l'enjeu du procès change dès lors qu'il implique des Biscayens solidement intégrés. L'accusation de fabrication de fausse monnaie, abandonnée par la suite au profit de celle de contrebande de fausse monnaie, donnait au corregidor de Bilbao l'occasion d'élever l'affaire vers un éventuel cas de lèse-majesté ${ }^{31}$, et de se libérer ainsi des contraintes imposées par les fors de la province. Mais le grand juge de Biscaye préfère toujours la prudence face à une junte générale qui, jalouse de ses prérogatives, défend avec acharnement le statut fiscal et juridique de la province. Cette prudence a aussi des racines financières. Selon le licencié Castillo de Bobadilla ${ }^{32}$, conseiller de Castille et procureur de la chancellerie de Valladolid, 260000 des 300000 maravédis, qui constituent la rente annuelle du corregimiento de Bilbao et de la seigneurie de Biscaye à la fin du $\mathrm{XVI}^{\mathrm{e}}$ siècle, dépendent directement de la province et donc de ses autorités forales.

\section{Le déploiement frustré de la majesté}

17 Cette défense forale restreint le déploiement de la majesté judiciaire en Biscaye. Par exemple, en 1674, le Sindico procurador, auquel incombe la défense des fors de Biscaye quand la junte générale n'est pas réunie, réclame une amende de 2000 ducats au greffier Santiago de la Toba pour avoir transmis au conseil royal les arrêts originaux d'une enquête pour fausse monnaie ${ }^{33}$. Santiago de la Toba est accusé de contrevenir aux fors de Biscaye et de violer l'autorité de la junte provinciale, et pour mieux le stigmatiser, Lopez 
de San Martín en fait un fraudeur. Il l'accuse aussi d'abus dans les sommes perçues pour les différentes écritures rédigées au détriment des parties. La vénalité supposée de Santiago de la Toba, qui l'aurait conduit à cacher aux parties en litige certaines pièces du procès pour leur soutirer des frais indus, vient renforcer le scandale de son attitude. Lors de plusieurs voyages à la cour, il aurait emporté non seulement les arrêts du procès en fausse monnaie mais aussi d'autres papiers sur des procédures civiles concernant des Biscayens, qui voient là leur droit d'être jugés par les leurs bafoué. Pour le procureur de la Biscaye, il n'y a pas de cas réservé qui permettrait de se soustraire à la juridiction seigneuriale de la Biscaye. Le 19 juin 1676, Santiago de la Toba (qui est aussi le greffier de la ville de Bilbao) se défend en affirmant qu'il n'avait pas à rendre compte de cette affaire à la junte, et que seul le grand juge est compétent pour contester cette transmission au conseil royal. Par ailleurs il contredit le procureur en rappelant qu'il est des cas d'exception qui relèvent directement de la majesté et non des fors de Biscaye. Charles II intervient pour rappeler les différentes commissions octroyées à Santiago de Toba et établir la légalité de ses agissements. Le conseil de Castille finit par statuer sur son cas et relève la légalité de toutes les sommes perçues, y compris celles issues de la vente des biens des faux-monnayeurs, que le procureur voulait récupérer.

Les coups de majesté, plus aptes à porter la stigmatisation du crime de monnaie sur l'étranger au royaume, doivent donc venir «directement » de Madrid. Dès juillet 1624, Philippe IV et son conseil de Castille réagissent avec fermeté, envoyant le grand juge de la cour de Navarre, Pedro de Alarcón de Ocón, mener la chasse à la criminalité monétaire en Guipuzcoa, avec les pouvoirs de haute justice d'un juge de commission ${ }^{34}$. Usant de la torture, il procéda contre des habitants de Saint-Sébastien et de Tolosa, allant jusqu'à incriminer l'officier des douanes (alcalde de sacas) d'intelligence avec l'étranger. Selon lui, la fausse monnaie de billon des Hollandais était transportée à partir des flottes de La Rochelle, Bordeaux, Bayonne et Saint-Jean-de-Luz qui faisaient le commerce des laines et du poisson avec Irún et Saint-Sébastien. Ce premier coup de force est suivi d'un second. Le licencié Geronimo de Avellaneda Manrique, à la chancellerie de Valladolid, reçoit du roi une commission extraordinaire, sous le contrôle du conseil de Castille, pour inspecter les villes de Irun, Fontarabie, El Pasaje, Saint-Sébastien, Orio, Zarauz, Guetaria, Zumaya, Deba, Motrico, Alcola, Aondarva, Lequeitio, Mundaca, Anchova, Bermeo, Portugalete, Bilbao et Guernica. L'ensemble du littoral de la Biscaye et du Guipúzcoa est concerné. Le 12 juillet 1627, les marchands de Saint-Sébastien, Pedro Fernández Pajo, Melchor Nuñez Peña, Luis de Oliva y Usto se plaignent auprès du conseil de Castille parce que le commissaire Avellaneda a fait saisir leurs marchandises débarquées de Hambourg et de France sur les quais du port basque sous le prétexte qu'elles servaient à cacher l'introduction de fausse monnaie ${ }^{35}$. Ils lui reprochent d'abuser de sa commission pour placer sous séquestre d'autres cargaisons (citrons, tabac, toile). Dès le 26 août 1627, Philippe IV saisit le conseil de Castille sur cette question. La relation des fautes, des fraudes effectuées par les accusés, que rédige Avellaneda qui leur reproche de sortir illégalement du royaume la monnaie d'or et d'argent, met fin à ce recours. Pedro Fernández, dont la justice se rappelle alors la nationalité portugaise, est toujours emprisonné en 1629.

19 C'est sur commission privative qu'en 1669, le juge municipal de Bilbao, le capitaine Juan de Barraycua, peut instruire un procès pour faux monnayage, dont les pièces seront transmises en appel directement au conseil de Castille, cour suprême de justice du royaume ${ }^{36}$. Cette affaire de faux monnayage commence par l'arrestation d'un Français de 
Bayonne, Pedro de Ugaz, dont les biens sont confisqués. Son emprisonnement permet l'arrestation de ses supposés complices. L'un d'entre eux, le capitaine Juan de Montano, est interrogé dès le 9 décembre 1669. Échevin du conseil de San Jurce, il reconnaît fréquenter les autres complices de l'entreprise criminelle, Juan de Zecanda, Martín de Cehabarria, Joseph de Visategui, Domingo de Larrave et le prisonnier Pedro de Ugaz. Dans l'interrogatoire qu'il mène, Juan de Barraycua insiste sur le rôle supposé de toutes ces personnes dans la fabrication de fausse monnaie et dans sa diffusion sur la côte espagnole à partir des ports français d'Hendaye et de Bayonne. L'accusation portée par Juan de Barraycua se fait ensuite plus précise : des maisons serviraient de dépôt à Bilbao et dans ses environs, c'est-à-dire dans des lieux dépeuplés où vivraient des maîtres artisans français, fabricateurs de fausse monnaie et bien équipés en marteaux et en faux sceaux du roi catholique. Les campagnes basques seraient envahies par des faux-monnayeurs français. En réalité, au moment où l'alcade de Bilbao interroge Juan de Montano, plusieurs perquisitions de ses auxiliaires ont permis de découvrir dans la maison de Juan de Otaola une grande quantité de faux doublons et dans celle de Concepción de Goyareu un millier de faux réaux. Les maisons de Mariana de Llona sur la Ribera del coronal de la ville de Bilbao sont elles aussi inspectées dès le 16 octobre 1669. Les gens de l'alcade ordinaire y trouvent 69 pains de goudron et des ouvrages de métal. L'accusé, Juan de Montano, nie les faits qui lui sont reprochés mais finit par avouer qu'il s'est rendu chez Domingo de Orma pour lui remettre du sucre et du plomb, après que ce dernier eut conclu avec un certain Dubrocart la vente de bois et d'une embarcation. Un autre témoin, Domingo de Zalduamaguregui, vezino de Bilbao, est interrogé le 20 décembre suivant pour savoir si le français Pedro de Ugaz vient régulièrement à Bilbao depuis 1668 et s'il est bien maître de pinasse à Bayonne.

Parallèlement, dès 1670, une deuxième procédure pénale est ouverte par l'autre alcade de Bilbao, le capitaine Pablo de Alçueta y Bertiz, à l'encontre de Juan de Otoala y Ruchi, Martin de Echabarria, Joseph de Ussategui, Juan de Zecanda et Domingo de Larrave, tous en fuite. Les autorités judiciaires font traduire du français au castillan, les livres et papiers saisis chez Juan de Otaola. C'est à partir de cette découverte que sont réalisés de nouveaux interrogatoires et de nouvelles arrestations. Concepción de Goyarens, âgée de 34 ans, est assignée à sa résidence, rue Tenderia à Bilbao, et prétend ne pas savoir lire ses propres livres de comptes, qui ont été saisis avec l'ensemble de ses biens. Ces livres de comptes sont ensuite vérifiés, amenant les enquêteurs à interroger Damian de Guendica, Pedro de Zavalaorue, ainsi que deux Anglais, Ricardo Aclan et Nicolas Chines, qui exhibent leurs propres livres de compte pour montrer qu'ils concordent avec ceux de Concepción de Goyarens. Les Anglais déclarent que l'accusée leur doit 6076 réaux de billon et 40 réaux d'argent. Une dernière relation, Bartolomé Baqur, déclare avoir reçu de Concepción de Goyarens pour paiement d'une dette, la somme de 19032 réaux d'argent qu'il a inscrite dans son livre, et rajoute qu'elle doit encore 2953 réaux d'argent à son frère. Ils n'en sont pas moins suspectés de complicité et leur maison est placée sous la surveillance de deux gardes. L'emprisonnement de Concepción de Goyarens a fait grand bruit et de nombreux créanciers viennent désormais réclamer leur dû auprès du juge.

21 L'affaire de fausse monnaie connaît un nouveau rebondissement avec l'emprisonnement d'un facteur, Domingo Larrave. Âgé de 25 ans, il est installé comme marchand à Nájera avec un certain Francisco. Le juge le soupçonne de s'être rendu dans cette ville le 14 août 1669, après deux jours de voyage, et d'y avoir résidé trois mois dans un couvent franciscain sous la protection du frère Miguel de la Guerta, avant de revenir de nuit à 
Bilbao, où il a été intercepté alors qu'il voulait ajuster ses comptes avec ses correspondants. Il aurait été prévenu de l'évolution de la procédure pénale par le courrier d'un de ses complices. Domingo Larrave nie ces accusations mais reconnaît s'être rendu à Bayonne pour recevoir de Juan de Leranda cinq quintaux de métal et une pinasse. Mais les lettres saisies sur sa personne montreraient que l'accusé aurait demandé à son frère, Juan de Larrave, un franciscain, de conserver un objet dans son couvent à Nájera. Les nouveaux éléments matériels de l'affaire entraînent l'interrogatoire de nouveaux témoins, dont l'épouse du prévenu, Maria de Ecorriaga, qui le 2 février 1670, déclare savoir lire et écrire, avant d'avouer que la lettre retrouvée sur la personne de son mari est bien celle de Juan de Larrave, comme le livre de compte saisi. Un autre témoin, Pedro de Abizuvez, reconnaît que l'accusé s'est bien enfui pendant la nuit en 1669, en lui disant que la justice avait procédé à plusieurs arrestations pour faux monnayage. Un autre témoin, alguazil de Bilbao, Paschoal de Ynchaurraga, confirme ces dires et reconnaît que Domingo Larrave a fait irruption dans sa chambre le soir à $7 \mathrm{~h} 30$ avant de fuir la ville. À la suite de ces nouveaux témoignages, le juge décide de réinterroger Domingo Larrave. Son interrogatoire nous montre que le magistrat pense qu'il existe une troisième place de faux-monnayeurs, Saint-Jean-de-Luz, et que l'accusé est retourné clandestinement à Bayonne sur un navire français.

La chronique de cette procédure pénale révèle la présence d'un portrait judiciaire du Français faux-monnayeur. La fausse monnaie est toujours française. Même si elle est produite dans l'arrière-pays basque, elle demeure française car les matériaux nécessaires à cette entreprise sont supposés français de fabrication et achetés à Bayonne. Le procès de Bilbao insiste sur le rôle des marchands et des artisans français. C'est d'ailleurs et d'abord un Français que l'on arrête, laissant le temps de fuir à ses complices locaux. L'enquête pourtant ne prouve pas que l'entreprise soit dirigée par des Français, malgré l'insistance du juge à faire de Bayonne, Saint-Jean-de-Luz et Hendaye le cœur du réseau criminel. Ce réseau utilise d'ailleurs les routes commerciales terriennes et maritimes de la frontière française comme il en utilise les hommes. Les différents témoignages contenus dans les actes de ce procès montrent en effet de larges complicités locales. Certains officiers locaux comme un alguazil sont indirectement compromis. Un couvent franciscain est accusé de protéger les accusés et de faire le jeu de l'étranger.

Nous ne pouvons que constater l'intégration sociale du faux-monnayeur, dont l'entreprise collective est fort bien implantée dans la société locale. C'est là sans doute ce qui justifie le coup de majesté, seul capable de réprimer les faux-monnayeurs français lorsqu'ils sont soutenus par une partie de la société basque.

$\mathrm{Du} \mathrm{XVI}^{\mathrm{e}}$ au $\mathrm{XVII}^{\mathrm{e}}$ siècle,la monarchie hispanique attache la criminalité monétaire à la notion de frontière, tant sa politique monétaire relève de l'exercice de sa souveraineté, dans un contexte guerrier de plus en plus affirmé. De ce fait, les provinces basques sont appelées à représenter le paradigme de cette réalité, aussi bien dans l'esprit des conseillers du roi et de l'administration judiciaire qui en dépend, que dans l'abondante littérature des donneurs d'avis et la pensée politique de la décadence espagnole. Le faux monétaire devient, dans ce contexte, constitutif de la guerre, et dès le début du XVII siècle avec les faux billons, une véritable arme de guerre entre des puissances hostiles, même lorsqu'elles respectent une bonne entente apparente entre elles. C'est par exemple 
le cas entre le roi catholique et les rebelles hollandais pendant la trêve des douze ans (1609 à 1621) ou entre Madrid et Paris après la paix de Vervins, de 1598 à 1635 . Le principe de défense de la monarchie catholique qui en découle, assimilé à la défense de la monnaie comme l'expression de la «souveraineté » du roi catholique, fait de l'ensemble des violations du ius monetae l'un des éléments moteurs de la fabrique d'une police des étrangers, non sans impliquer des conflits entre les différentes autorités pour accaparer ce nouveau champ d'action, entre la cour et les provinces basques attachées à leur statut fiscal et à leurs intérêts commerciaux.

Les résistances sont constantes car les élites politiques locales investissent massivement dans le commerce de contrebande ${ }^{37}$. Il n'en demeure pas moins que s'affirme un lien entre la criminalité monétaire des étrangers et les réseaux traditionnels de la contrebande ${ }^{38}$. La contrebande monétaire utilise les mêmes réseaux et les mêmes routes maritimes et littorales ; elle implique des lieux interlopes comme les ports atlantiques de Bilbao, SaintSébastien, Irun, Hendaye, Saint-Jean-de-Luz, Bayonne et Nantes.

L'intégration de cette économie de l'illicite dans la société basque comme la résistance de la junte de Biscaye à l'application des mesures demandées par la monarchie au corregidor de Bilbao, oblige le conseil de Castille à promouvoir de nouvelles procédures judiciaires pour y affirmer son influence et développer des «coups de majesté » inachevés plus qu'une réelle politique de répression continue des étrangers criminels de monnaie. Le déploiement de la justice souveraine du conseil de Castille par l'envoi de commissaires dotés d'une juridiction privative d'exception ou par la reconnaissance d'une délégation particulière à un juge local étroitement contrôlé depuis Madrid, ne suffit pas systématiquement à déplacer le crime monétaire vers la lèse-majesté, c'est-à-dire la justice réservée du seul monarque. Néanmoins, il s'agit là d'événements qui fonctionnent comme autant de signes témoignant de la suprématie symbolique de l'autorité royale. Plus encore qu'un objet de répression, le faux monétaire représente, au sein d'une criminalité de monnaie bien plus ample, un vecteur de connaissance gouvernementale sur les communautés étrangères établies dans les ports atlantiques. Répressions judiciaires, résistances forales et sociales, luttes des pouvoirs locaux et de la cour madrilène, la bataille pour asseoir le pouvoir monétaire de la monarchie catholique instrumentalise la police des étrangers. En ce sens, l'expérience hispanique et atlantique du crime de monnaie des étrangers conduit l'expérience de l'« État monarchique ».

\section{NOTES DE FIN}

1. Esteban EstRíngana, Alicia, Guerra y finanzas en los Paises Bajos católicos. De Farnesio a Spinola (1592-1630), Madrid, Laberinto, 2002.

2. LAPEYRE, Henri, El comercio exterior de Castilla a traves de las aduanas de Felipe II, Valladolid, Universidad de Valladolid, 1981 ; PRIOTTI, Jean Philippe et SAUPIN, Guy (dir.), Le commerce atlantique franco-espagnol. Acteurs, négoces et ports ( $\mathrm{XV}^{e}$-XVIII ${ }^{e}$ siècles), Rennes, PUR, 2008.

3. SANTIAgo FeRnÁndeZ, Javier de, Política monetaria en Castilla durante el siglo XVII, Valladolid, Junta de Castilla y León, 2000. 
4. VILAR, Pierre, Or et monnaie dans l'Histoire, Paris, Flammarion, 1974, p. 295.

5. Froidevaux, Ch., Clairand, A., « Faux monnayage et crises monétaires sous l'Ancien Régime ", AUBERSON, Anne Francine, DRESCKA, Harald R. et FREY-KUPPER, Suzanne (dir.), Faux-contrefaçons-imitations. Actes du quatrième colloque international du Groupe suisse pour l'étude des trouvailles monétaires (Martigny, $1^{\text {er }}-2$ mars 2002), Lausanne, Le Zèbre, 2004, p. 175-234.

6. GIRARD, Albert, Le Commerce français à Séville et Cadix au temps des Habsbourg, Paris, 1932, p. 439-440.

7. BRAUDEL, Fernand, La Méditerranée et le monde méditerranéen à l'époque de Philippe II, Paris, A. Colin, réed. 1990 (1966), t. I, p. 488-489 ; GELABERT, Juan E., La bolsa del rey. Rey, reino y fisco en Castilla (1598-1648), Barcelona, Crítica, 1997, p. 134 ; CARRASCo VÁzQUEZ, Jesús, «Contrabando, moneda y espionaje (el negocio del vellón : 1606-1620) », Hispania, 1997, LVII/3, n 197, p. 1081-1105.

8. SANTIAGo FERnÁNDEZ, Javier de, El arbitrio monetario de Pedro de Oña (1607). Edición y estudio crítico, Madrid, Castellum, 2002, p. 99.

9. MONCADA, Sancho de, Restauración politica de España, Madrid, Instituto de Estudios Fiscales, 1974, p. 163.

10. GARCía Guerra, Elena María, Moneda y arbitrios consideraciones del siglo XVII, Madrid, CSIC, 2003, p. 89-94.

11. Archivo General de Simancas, e, Francia, Legajo K-1427, fo 135.

12. García García, Bernardo José, La Pax Hispánica. Política exterior del Duque de Lerma, Leuven, Leuven University Press, 1996, p. 198 et 356.

13. GonZÁlez PALENCIA, Ángel, La Junta de reformación 1618-1623, Valladolid, Poncelix, 1932, p. 272-273.

14. Paz Alonso, María, El proceso penal en Castilla (siglos XIII-XVIII), Salamanca, Universidad de Salamanca, 1982, p. 303 et 304.

15. Sur la question de l'étranger, voir DomínguEz ORTíz, Antonio, Los Extranjeros en la vida española durante el siglo XVIIy otros artículos, Sevilla, Diputación de Sevilla, reed. 1996 ; Les Français en Espagne à l'époque moderne (XVI ${ }^{e}$-XVIII ${ }^{e}$ siècles), Paris, CNRS, 1990 ; VILLAR GARCíA, M. B., PEZZi CRISTÓBAL. P. (eds.), Los Extranjeros en la España moderna, Málaga, Universidad de Malaga, 2003 ; GARCíA GARCíA Bernardo José (pres.), La Monarquía de las naciones. Patria, nación y naturaleza en la Monarquía de España, Madrid, Fundación de Amberes, 2004 ; C APOROSSI, Olivier, « Le statut des Anglais dans l'Espagne de Philippe IV (1621-1665) », Anglophonia. French Journal of English Studies, 2005, n 17, p. 163-173.

16. MARTín RodRíGUEZ, Jacinto, " Figura histórico-jurídica del Juez Mayor de Vizcaya », Anuario de Historia del Derecho Español, 1968, n 38, p. 641-669.

17. BeRMEJO CABRERo, José Luis, Poder político y administración de justicia en la España de los Austrias, Madrid, Ministerio de Justicia, 2005, p. 90.

18. Compétent en matière civile et pénale, le grand juge de Biscaye reçoit en appel les décisions de toutes les justices de la seigneurie. Au vu des sources judiciaires consultées, il faut donc distinguer au moins trois échelles juridictionnelles : la municipalité, le corregidor et la seigneurie. Ces deux dernières juridictions, bien qu'assumées par la même personne n'en demeurent pas moins distincte l'une de l'autre. Les sentences rendues par le grand juge peuvent être contestées devant le juge des requêtes de Biscaye ( juez de las suplicaciones de Vizcaya), dont la charge relève directement du président de la chancellerie de Valladolid, et qui a autorité pour traiter lui-même l'affaire ou la déléguer à une des chambres des auditeurs de la dite chancellerie. HERAS SANTOS, José Luis de las, 
La Justicia penal de los Austrias en la corona de Castilla, Salamanca, Universidad de Salamanca, 1994, p. 72.

19. Archivo de la Real Chancilleria de Valladolid, Sala de Vizcaya, Pleitos, Caja 2409, ex. 3 (2409/0003).

20. Archivo de la Real Chancilleria de Valladolid, Sala de Vizcaya, Pleitos, Caja 1154, ex. 4.

21. GIRARD René, Le Bouc émissaire, Paris, réed. Livre de Poche, 2006 (1982), p. 28-31.

22. Archivo de la Real Chancilleria de Valladolid, Sala de Vizcaya, Pleitos, Caja 1760, ex. 1.

23. Archivo de la Real Chancilleria de Valladolid, Sala de Vizcaya, Pleitos, Caja 1723, ex. 2.

24. Archivo de la Real Chancilleria de Valladolid, Sala de Vizcaya, Pleitos, Caja 570, ex. 1.

25. GARCíA HERNAN, Enrique, Irlanda y el rey prudente, Madrid, Hermes, 2000, p. 209-246.

26. Ibidem, p. 208.

27. Juntas y regimientos de Bizkaia. Actas de Villas y Ciudad, t. III, Agosto 1589-1600, Juntas generales de Bizkaia, 1999, p. 594-595.

28. Archivo de la Real Chancilleria de Valladolid, Sala de Vizcaya, Pleitos, Caja 516, ex. 3.

29. Archivo de la Real Chancilleria de Valladolid, Sala de Vizcaya, Pleitos, Legajo 2113, ex. 5 .

30. Archivo de la Real Chancilleria de Valladolid, Sala de Vizcaya, Pleitos, Caja 1228, ex. 2.

31. Pradilla, Francisco de la, Suma de las leyes, Madrid, Imprenta real, 1644, p. 14.

32. CASTILlO DE BOVADILla, Jeronimo, Política para corregidores y señores de vasallos, en tiempo de paz, y de guerra, Amberes, Juan Bautista Verdussen, reed. 1704, t. II, p. 647.

33. Archivo de la Real Chancilleria de Valladolid, Sala de Vizcaya, Pleitos, Caja 2409, ex. 3.

34. TRUCHUElo García, Susana, Gipuzkoa y el poder real en la Alta Edad Moderna, San

Sebastián, Diputación Foral de Gipuzkoa, 2004, p. 463.

35. Archivo Histórico Nacional, Consejos, Legajo 29616, ex. 11.

36. Archivo Histórico Nacional, Consejos, Legajo 25925, ex. 4.

37. C'est le cas, par exemple, d'Ochoa de Otañes Salazar (1598-1653) qui, familier de l'Inquisition depuis 1633, occupe à plusieurs reprises les fonctions d'échevin de Portugalete entre 1620 et 1648 , ainsi que celles d'alcade (juge local) entre 1628 et 1653, et de Sindico Procurador General entre 1639 et 1651, cette dernière charge lui permettant de défendre devant la junte générale de Biscaye la réforme des ordonnances royales de 1615 sur le trafic du vin dont il faisait la contrebande depuis au moins 1646. Ochoa de Otañes investit dans diverses activités, licites et illicites, touchant aussi bien le trafic maritime avec Lisbonne et les Flandres, que la course, le commerce du sel, du fer ou la vente des navires, développant des échanges avec les pays ennemis de l'Espagne ou en révolte contre le roi catholique. PÉREZ HERNÁNDEZ, Santiago, « Un familiar del Santo Oficio en un puerto vasco durante la primera mitad del siglo XVII. Ochoa de Otañes en su comunidad », Revista de la Inquisición, 2001, n 10, p. 283-333.

38. Pour une synthèse sur les réseaux de la contrebande dans l'espace atlantique, $M$ ANTECón MOVELLÁN, Tomás A., « Les réseaux de contrebandiers dans les ports atlantiques de Castille au cours du XVII ${ }^{\mathrm{e}}$ siècle », SAUPIN, Guy (dir.), Villes atlantiques dans l'Europe occidentale du Moyen Âge au XXe siècle, Rennes, PUR, 2006, p. 315-335 ; AllozA APARICIO, Ángel, Europa en el mercado español. Mercaderes, represalias y contrabando en el siglo XVII, Salamanca, Junta de Castilla y León, 2006. 


\section{RÉSUMÉS}

Entre 1551 et 1700, dans un contexte guerrier de plus en plus affirmé, la monarchie hispanique rattache la criminalité monétaire à la notion de frontière. Attachée à l'exercice de sa souveraineté, elle voit dans les provinces basques (et notamment dans la seigneurie de Biscaye) le laboratoire d'une nouvelle répression des crimes de monnaie, tournée vers la police des étrangers et vers l'affirmation de la majesté du roi catholique. L'étude des sources judiciaires et administratives montre les difficultés et les résistances qu'une telle politique peut susciter dans les sociétés littorales de l'Espagne atlantique.

Between 1551 and 1700 in a military context, the Hispanic monarchy linked crime of money to the notion of border. Attached to the exercise of its sovereignty, the crown saw in the Basque Provinces (especially in the Lordship of Biscay) the laboratory of a new crackdown on crimes of money, focused on the aliens police and the affirmation of majesty. The study of judicial and administrative sources shows the difficulties and resistance that such policy may generate in the coastal societies of Spain Atlantic.

\section{INDEX}

Index chronologique : XVIIIe siècle, XVIIe siècle, XVIe siècle

Thèmes : Espagne

\section{AUTEUR}

\section{OLIVIER CAPOROSSI}

Maître de conférences en histoire moderne université de Pau et des Pays de l'Adour FRAMESPA UMR 5136 